\title{
EFEITOS MICROCLIMÁTICOS DE UM FRAGMENTO DE FLORESTA URBANA EM CURITIBA-PR
}

\author{
MICROCLIMATIC EFFECTS OF AN URBAN FOREST FRAGMENT IN \\ CURITIBA-PR
}

\author{
Eduardo Praxedes Bomfim Raymundo', Daniela Biondi², Fernando Vicente Dacól3, \\ Mauricio da Silva Leal ${ }^{4}$, Kelvyn Reksidler ${ }^{5}$
}

\section{RESUMO}

A urbanização limitou demasiadamente os remanescentes vegetais, aumentando a importância dos fragmentos florestais na atenuação do microclima local. Objetivou-se analisar a influência microclimática de um fragmento de floresta urbana (Capão do Tigre, localizado no campus III - Jardim Botânico da Universidade Federal do Paraná) em todas as estações do ano. Foram obtidos dados de velocidade do vento, temperatura do ar e umidade relativa do ar. Para isso, foi utilizado três miniestações micrometeorológicas portáteis da marca Kestreß, modelo 4.200, duas permaneceram estáticas no centro e na borda florestal, e a outra fez o percurso de 6 pontos distribuídos ao longo do estacionamento, no período entre $11 \mathrm{~h}$ e $14 \mathrm{~h} 30$, simultaneamente, pois este período cobre a faixa de maior incidência de radiação e consequentemente maior temperatura. As médias das variáveis observadas diferenciaram significativamente em todas as estações do ano. As mudanças mais acentuadas nas variáveis meteorológicas foram encontradas entre o centro do fragmento florestal e a área do estacionamento, sendo a borda do fragmento o local onde as médias se apresentaram intermediárias aos demais lugares. Conclui-se que o fragmento florestal exerce influência no microclima local (temperatura, umidade do ar e velocidade do vento), em todas as estações do ano.

Palavras-chave: Temperatura do ar; Umidade relativa do ar; Velocidade do vento; Conforto térmico; Áreas verdes.

\begin{abstract}
Urbanization overly limited the vegetation remnants, raising the importance of forest fragments in attenuating local microclimates. The objective of this study was to analyze the microclimate influence of a fragment of urban forest (Capão do Tigre, located on campus III of the Federal University of Paraná) in all seasons of the year. Data on wind speed, air temperature, and relative humidity were recorded. For this, three micrometeorological portable stations of the brand Kestrel ${ }^{\circledR}$, model 4,200 , were used, two permanent placed at the center and the edge, and another made the tracking of 6 points distributed along the parking lot, during the period of $11: 00 \mathrm{am}$ and 2:30 pm, as this period covers the range of greater radiation incidence and consequently higher temperature. The average of the observed variables differs in all seasons. The most accentuated changes in the meteorological variables were found between the center of the forest fragment and the parking area, and the fragment's edge is the place where averages are described as intermediaries for other places. It is possible to conclude that the forest fragment influences the local microclimate (temperature, humidity, and wind speed), in all seasons of the year.
\end{abstract}

Keywords: Air temperature; Relative humidity; Wind speed; Thermal comfort; Green areas.

Recebido em 06.04.2020 e aceito em 07.07.2020

1 Graduando em Engenharia florestal. Universidade Federal do Paraná. Curitiba/PR. Email: edupraxedess@gmail.com

2 Engenheira Florestal. Profa. Dra. Universidade Federal do Paraná. Curitiba/PR. Email: danielabiondibatista@gmail.com

3 Graduando em Engenharia florestal. Universidade Federal do Paraná. Curitiba/PR. Email: fernando.dacol@gmail.com

4 Graduando em Engenharia florestal. Universidade Federal do Paraná. Curitiba/PR. Email: mauricio.leal.silva@hotmail.com

5 Graduando em Engenharia florestal. Universidade Federal do Paraná. Curitiba/PR. Email: kelv132@hotmail.com 


\section{INTRODUÇÃO}

Na segunda metade do século XX o processo de urbanização acentuou-se cada vez mais, primeiro com um intensivo aumento do número de núcleos populacionais e em seguida uma multiplicação das cidades e suas respectivas regiões metropolitanas (SANTOS, 2008). Esse longo processo de ocupação de terras, no qual a vegetação fragmentou-se em pequenas porções de mata nativa, incluindo as denominadas de fragmentos florestais urbanos, que são de extrema importância social, política, econômica e ecológica, portanto, devem ser preservados (GUIRAO; FILHO, 2011).

A vegetação urbana quando bem conservada, pode cumprir diversas funções, assim como Bargos e Matias (2011) explanaram: Social - pela possibilidade de lazer, como um refúgio da área de intenso fluxo de pessoas; Estética - na diversificação da paisagem construída e embelezamento da cidade; Educativa - proporciona um excelente ambiente para atividades extraclasse e projetos de educação ambiental; Psicológica - proporciona um contato com a natureza e faz com que a sociedade tenha relaxamento tanto físico quanto psicológico; e Ecológica - no provimento da filtração do ar, melhorias na qualidade da água e do solo, manutenção da fauna, infiltração d/água e melhora no clima local, resultando no bem-estar dos habitantes.

Entretanto, Kudo, Pereira e Silva (2016) afirmam que "os fragmentos florestais urbanos são tratados como tipos genéricos de espaços protegidos para os quais não são previstos mecanismos operacionais de delimitação e regime especial de proteção e de gestão pública."

Dentre todas as transformações ocorridas, a supressão das áreas verdes foi inevitável no crescimento desenfreado das cidades, esse espaço construído altera os elementos meteorológicos, formando diferentes microclimas (FEITOSA, 2011). A manutenção desses fragmentos é de extrema importância para a obtenção de melhores condições de conforto térmico, promovendo uma maior qualidade de vida para a população (MARTINI; BIONDI, 2015).

Com o passar do tempo notou-se que, quando a quantidade de edificações é elevada menos favorável climaticamente o local se torna ao homem, assim como acontece no meio urbano onde os elementos climáticos temperatura, umidade e o movimento do ar são afetados consideravelmente (BARBIRATO; SOUZA; TORRES, 2007).

Esses elementos atuam de forma conjunta, formando um microclima local, que no caso dos grandes centros urbanos desencadeiam um fenômeno chamado de "ilha de calor", caracterizado pela elevação da temperatura, tal fato ocorre devido à alta densidade de materiais absorventes da radiação solar - cimento, asfalto, concreto e aço - os quais emitem essa radiação novamente na forma de calor (SAMPAIO; FARIA, 2014). 
Com isso, a vegetação, dentre todos os elementos urbanos, é o elemento mais versátil em relação a modificação climática, sendo útil para controlar a troca de radiação, fluxo de ar, ventilação de poluentes do ar, evaporação, temperatura, erosão, escoamento e ruído (OKE et al., 2017). Sendo assim, "a vegetação, enquanto fator climático" possui extrema relevância dentro do espaço urbano, pois age como um filtro de poluentes e gases atmosféricos, aumenta a permeabilidade do solo (ALVES; FIALHO, 2012) e atua diretamente no microclima urbano como um excelente controlador da temperatura e umidade do ar (BIONDI et al., 2011).

No caso da temperatura, as áreas verdes possuem o papel de atenuação, devido ao fechamento do dossel arbóreo que automaticamente gera um sombreamento específico, diminuindo assim a temperatura ambiente (MARTELLI; JR, 2015). Já a umidade do ar é maior dentro do espaço vegetado pelo fato da evapotranspiração, onde tanto a evaporação da água do solo quanto a transpiração foliar aumentam significativamente a taxa de vapor atmosférico (BARBIRATO; SOUZA; TORRES, 2007). Por fim a velocidade do vento, que segundo Tong et al. (2017) as árvores e edifícios atuam como barreiras para a fluidez do vento, portanto áreas abertas, sem bloqueio algum, facilita para que a velocidade do vento se intensifique.

Com base no exposto, o presente trabalho teve o objetivo de verificar a influência microclimática de um fragmento de floresta urbana na cidade de Curitiba - PR por meio do monitoramento das variáveis velocidade do vento, temperatura e umidade relativa do ar em todas as estações do ano.

\section{MATERIAL E MÉTODOS}

Este trabalho foi realizado em um fragmento de floresta conhecido como "Capão do Tigre", localizado no campus III - Jardim Botânico da Universidade Federal do Paraná em

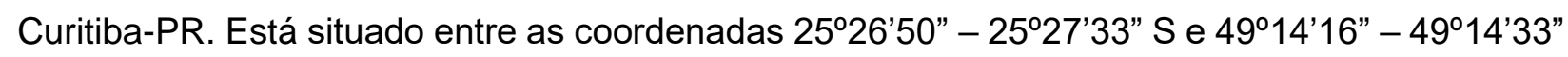
W, e a aproximadamente $900 \mathrm{~m}$ de altitude, com área estimada em $152.400 \mathrm{~m}^{2}$, sendo $85 \%$ da área de Floresta com Araucária (Floresta Ombrófila Mista Montana) e 15\% de capoeira, com predomínio de taquara (RONDON NETO et al., 2002).

O fragmento florestal estudado encontra-se entre duas rodovias federais (BR 116 e BR 277) e uma avenida de tráfego intenso (Avenida das Torres), além de bairros residenciais nas suas proximidades. Este fragmento possui solos Hidromórficos próximos aos canais de drenagem e Cambissolos e Podzólicos nas regiões drenadas, com relevo suavemente ondulado (RONDON NETO et al., 2002). Para Miguez (2001), o bairro Jardim Botânico, onde está inserido o fragmento de floresta (Capão do tigre), possui 6.153 habitantes em $2.772 .000 \mathrm{~m}^{2}$, onde $8,43 \%$ constitui-se de áreas verdes. 
De acordo com a classificação de Köppen, a região de Curitiba possui tipo climático Cfb, com temperatura média anual é $17,125^{\circ} \mathrm{C}$, variando aproximadamente $7,6^{\circ} \mathrm{C}$, com geadas e neve ocasional, e a pluviosidade média é $1390 \mathrm{~mm}$. (CLIMATE-DATA, 2010).

Os dados obtidos no levantamento meteorológico seguiram a metodologia de Leal et al. (2011), que define basicamente a diferença microclimática dentro e fora de um fragmento. Foram utilizadas três miniestações meteorológicas da marca Kestre伊, modelo 4200, mantidas a 1,50 $\mathrm{m}$, registrando três elementos microclimáticos: temperatura, umidade do ar e velocidade do vento. Todas as miniestações permaneceram sob o cuidado de um pesquisador, não sendo acopladas em nenhum suporte, coletando dados a cada minuto, simultaneamente, em um período aproximado de 25 minutos, conforme a figura 1.

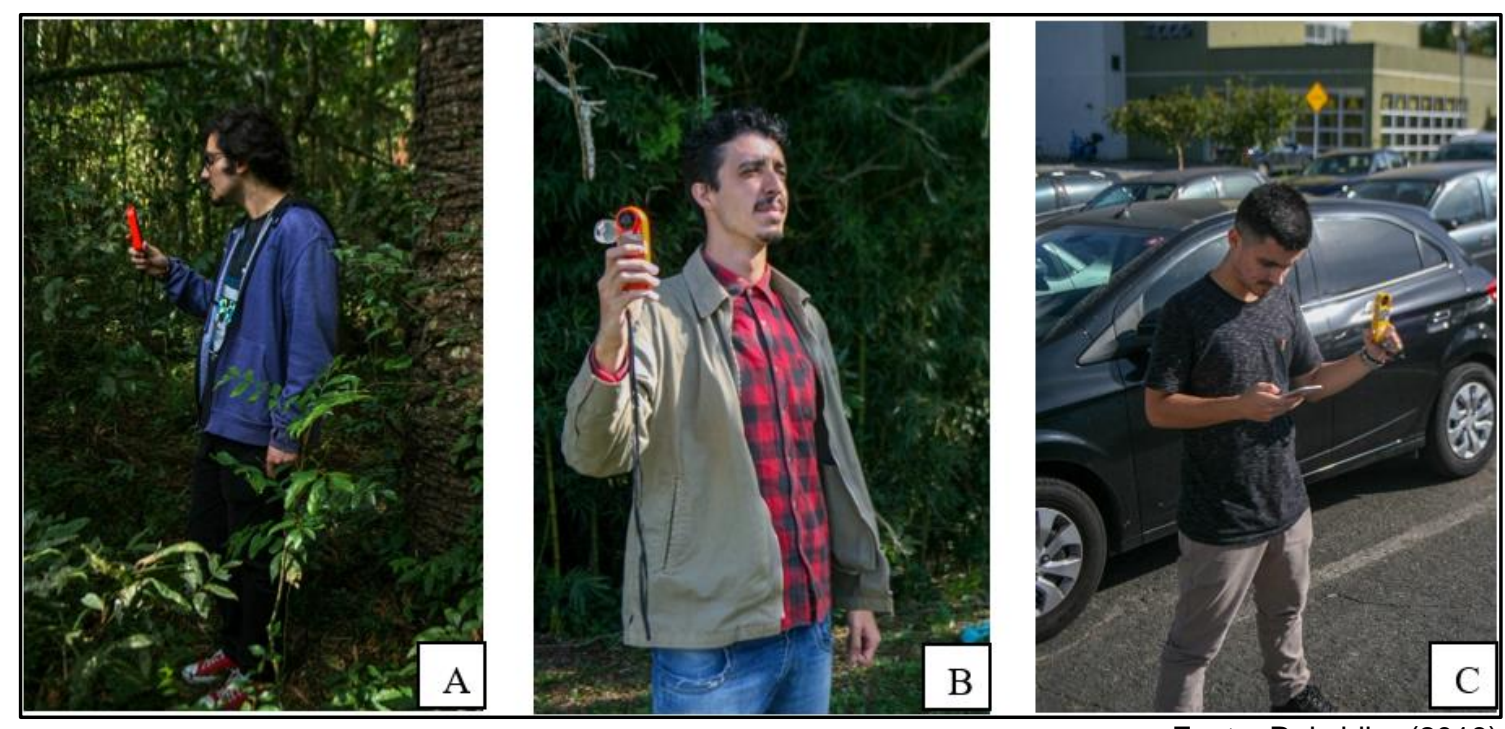

Fonte: Reksidler (2019)

Figura 1. Medição de temperatura do ar, umidade relativa do ar e velocidade do vento com miniestação meteorológica no centro do fragmento de floresta (A), na borda (B) e no estacionamento (C).

Figure 1. Temperature, relative air humidity, and wind speed measurement with the portable meteorological station at the center of the forest fragment (A), the edge (B), and the parking lot (C).

Duas miniestações permanecem imóveis, uma no centro e uma na bordadura do fragmento florestal. Já a outra fez todo o percurso do estacionamento, denominado como transecto móvel, cujo principal objetivo é minimizar os efeitos das diferentes intensidade de radiação produzidas pela variação da altura aparente do sol, evitando a necessidade de correção dos dados de acordo com o período de medição (LEAL et al. 2011), conforme apresentado na Figura 2. Esse trajeto consistiu em seis pontos de referência distribuídos ao longo do percurso, o qual foi feito quatro vezes (duas idas e duas voltas) para homogeneizar os dados. A escolha deste local, o estacionamento (local totalmente impermeabilizado por asfalto), foi devido à pouca ou nenhuma presença de árvores ao longo do transecto, servindo para análise comparativa. 


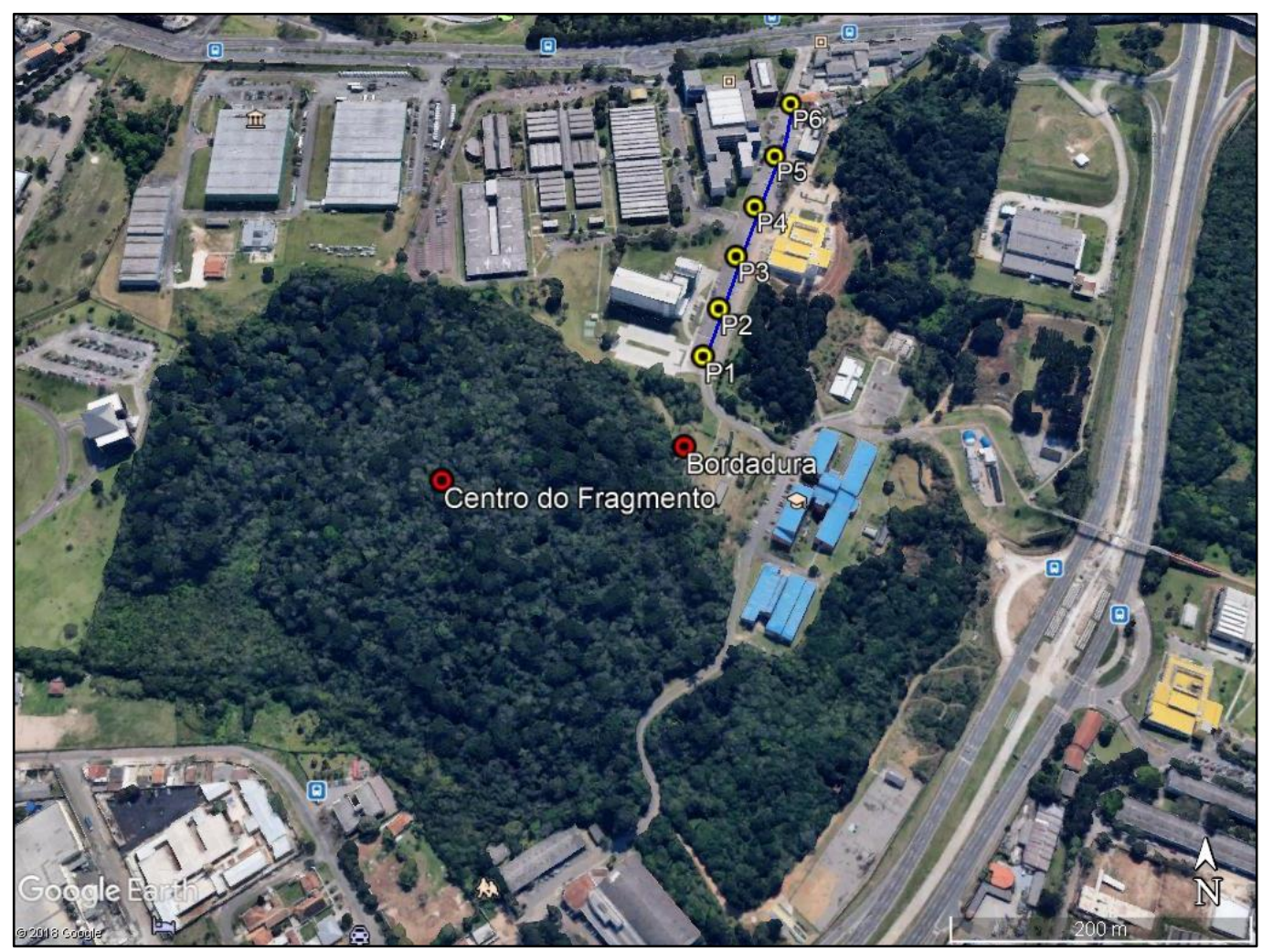

Fonte: Google (2018)

Figura 2. Localização geográfica dos pontos de coleta de dados.

Figure 2. Geographic localization of data collection points.

O período da coleta dos dados foi de aproximadamente um ano, tendo início na primavera de 2018 e o termino no inverno de 2019. Em cada estação do ano foram feitas três coletas, totalizando 12 coletas. A hora do dia destas coletas foi entre $11 \mathrm{~h}$ e $14 \mathrm{~h} 30$, pois este período cobre a faixa de maior incidência de radiação e consequentemente maior temperatura (SOARES; BATISTA; TETTO, 2015). Além disso, com a utilização desse período de coleta foi possível cobrir a variação do horário de verão ocorrida no sul do Brasil para o racionamento de energia. Assim como, as coletas foram feitas estritamente em dias de céu limpo, ou com menos de $50 \%$ do céu recoberto por nuvens altas, pois foi de extrema importância diminuir ao máximo o sombreamento proporcionado pelas nuvens no local observado.

Os dados adquiridos, com as miniestações coletando dados automaticamente a cada minuto, foram compostos por 24 repetições de cada variável meteorológica em todos os locais observados, totalizando 864 dados coletados em todo o período da pesquisa (12 meses), sendo esses alocados em forma de planilha. A partir disso, foram feitas médias de cada variável (temperatura, umidade do ar e velocidade do vento) em cada estação do ano, sendo aplicadas em um delineamento inteiramente casualizado e não sendo aplicada a análise de variância. As médias obtidas foram comparadas entre os ambientes em cada estação do ano, ao nível de 95\% de probabilidade pelo teste SNK. 


\section{RESULTADOS E DISCUSSÃO}

\section{Análise da temperatura do ar}

Os resultados obtidos em relação a variável meteorológica temperatura do ar demonstraram que existe diferença significativa entre as médias do estacionamento (ES), borda (BD) e o centro do fragmento de floresta (CFF) em todas as estações do ano (Tabela 1).

Tabela 1. Análise estatística da variável temperatura do ar em todas as estações do ano entre os locais estudados

Table 1. Statistical analysis of the air temperature variable in all seasons of the year among the locations studied

\begin{tabular}{cccc}
\hline \multirow{2}{*}{ Estação } & \multicolumn{3}{c}{ Temperatura do ar $\left({ }^{\circ} \mathbf{C}\right)$} \\
\cline { 2 - 4 } & ES & BD & CFF \\
\hline Primavera & $30,838 \mathbf{a}$ & $29,846 \mathbf{b}$ & $28,399 \mathbf{~}$ \\
\hline Verão & $31,113 \mathbf{a}$ & $29,921 \mathbf{b}$ & $28,017 \mathbf{c}$ \\
\hline Outono & $25,769 \mathbf{a}$ & $25,088 \mathbf{b}$ & $22,451 \mathbf{c}$ \\
\hline Inverno & $22,847 \mathbf{a}$ & $20,803 \mathbf{b}$ & $20,158 \mathbf{c}$ \\
\hline
\end{tabular}

Nota: Letras diferentes diferem estatisticamente pelo teste SNK na linha horizontal.

Legenda: $\mathrm{ES}=$ estacionamento, $\mathrm{BD}=$ borda do fragmento, $\mathrm{CFF}=$ centro do fragmento de floresta.

Em todas as estações do ano o estacionamento apresentou as maiores médias de temperatura, seguido da borda e, por último, o centro do fragmento florestal. Esse fato também ocorreu na pesquisa sobre conforto térmico efetuada por Martini e Biondi (2015) no Parque Municipal do Barigui em Curitiba - PR, no qual as temperaturas máximas registradas foram, no exterior do fragmento de floresta $24,1^{\circ} \mathrm{C}$, e no centro do fragmento florestal $19,8^{\circ} \mathrm{C}$, em média.

Isso ocorre devido ao sombreamento proporcionado pelas árvores e a diferença de características dos materiais (asfalto, cimento, edificações, etc.), fazendo com que, o ambiente sem vegetação emita mais calor à superfície (MARTELLI; JR, 2015).

Para Wang et al. (2015), na estação do verão, mais especificadamente em dias com céu limpo, o efeito de resfriamento proporcionado pelas árvores duplica, em relação a dias frios, onde o desempenho das árvores na mudança do microclima reduz consideravelmente.

Assim como Soldera, Batista e Martini (2014) em pesquisa realizada no mesmo local, verificou que a maior diferença de temperatura entre o interior e o exterior do fragmento é encontrada na estação do verão $\left(5,19^{\circ} \mathrm{C}\right)$, seguido da estação do outono $\left(4,57^{\circ} \mathrm{C}\right)$, inverno $(3,16$ $\left.{ }^{\circ} \mathrm{C}\right)$, e por último a estação da primavera $\left(2,86^{\circ} \mathrm{C}\right)$. 
Entretanto, não se verificou o mesmo no presente trabalho, pois a maior diferença de temperatura entre os locais observados foi na estação do outono $\left(3,31^{\circ} \mathrm{C}\right)$, seguido da estação do verão $\left(3,10^{\circ} \mathrm{C}\right)$, inverno $\left(2,69^{\circ} \mathrm{C}\right)$, e por último a estação da primavera $\left(2,44^{\circ} \mathrm{C}\right)$.

\section{Análise da umidade relativa do ar}

As médias da variável umidade relativa do ar foram maiores no centro do fragmento (CFF) e borda (BD) e menores no estacionamento (ES) (Tabela 2). Na estação da primavera a borda (BD) e o fragmento (CFF) não diferiram estatisticamente. No verão e no outono as médias foram maiores no centro (CFF), seguido da borda (BD) e, com as menores médias no estacionamento (ES). Na estação do inverno a maior média foi obtida na borda (BD), seguida do centro (CFF) e estacionamento (ES) (Tabela 2).

Tabela 2. Análise estatística da variável umidade em todas as estações do ano entre os locais estudados Table 2. Statistical analysis of the humidity variable in all seasons of the year among the locations studied

\begin{tabular}{cccc}
\hline \multirow{2}{*}{ Estação } & \multicolumn{3}{c}{ Umidade relativa (\%) } \\
\cline { 2 - 4 } & ES & BD & CFF \\
\hline Primavera & $44,555 \mathbf{~ b}$ & $50,497 \mathbf{a}$ & $51,085 \mathbf{a}$ \\
\hline Verão & $43,754 \mathbf{c}$ & $46,963 \mathbf{b}$ & $53,888 \mathbf{a}$ \\
\hline Outono & $48,853 \mathbf{c}$ & $52,572 \mathbf{~ b}$ & $62,435 \mathbf{a}$ \\
\hline Inverno & $53,342 \mathbf{c}$ & $61,685 \mathbf{a}$ & $58,385 \mathbf{~ b}$ \\
\hline
\end{tabular}

Nota: Letras diferentes diferem estatisticamente pelo teste SNK na linha horizontal.

Legenda: $\mathrm{ES}$ = estacionamento, $\mathrm{BD}$ = borda do fragmento, $\mathrm{CFF}=$ centro do fragmento de floresta.

Com isso, a umidade apresenta uma variação inversa da temperatura, valores maiores no interior do fragmento e borda e menores no estacionamento. Para Zhang, Lv e Pan (2013) a densidade da copa, a área da copa, a altura da árvore e a radiação solar são os quatro elementos essenciais para que essa relação inversa entre temperatura e umidade relativa do ar ocorra.

A maior diferença obtida foi de $13,5 \%$ na estação do outono, entre o centro do fragmento e o estacionamento. Bem como, em pesquisas similares a essa, Silva et al. (2014) no Bosque João Carlos Hartley Gutierrez em Curitiba - PR, com área de 35.000 m², os autores detectaram uma diferença de $17,8 \%$ entre o interior e o exterior do fragmento. Essa diferença significativa na umidade relativa do ar é resultante do contínuo processo da evapotranspiração, na qual tanto a evaporação quanto a transpiração atuam de forma conjunta (BARBIRATO; SOUZA; TORRES, 2007). 


\section{Análise da velocidade do vento}

No tocante a velocidade do vento, notou-se que as maiores médias foram obtidas no estacionamento (ES) em todas as estações do ano. Em relação ao centro do fragmento (CFF) e a borda (BD), foi verificado que somente na estação do outono as médias diferiram estatisticamente (Tabela 3).

Tabela 3. Análise estatística da variável velocidade do vento em todas as estações do ano entre os locais estudados

Table 3. Statistical analysis of the wind speed variable in all seasons of the year among the locations studied

\begin{tabular}{cccc}
\hline \multirow{2}{*}{ Estação } & \multicolumn{3}{c}{ Vento $(\mathbf{m} / \mathbf{s})$} \\
\cline { 2 - 4 } & ES & BD & CFF \\
\hline Primavera & $1,236 \mathbf{a}$ & $0,469 \mathbf{b}$ & $0,311 \mathbf{b}$ \\
\hline Verão & $1,771 \mathbf{a}$ & $0,529 \mathbf{b}$ & $0,283 \mathbf{b}$ \\
\hline Outono & $0,917 \mathbf{a}$ & $0,440 \mathbf{b}$ & $0,031 \mathbf{c}$ \\
\hline Inverno & $1,113 \mathbf{a}$ & $0,392 \mathbf{b}$ & $0,218 \mathbf{b}$ \\
\hline
\end{tabular}

Nota: Letras diferentes diferem estatisticamente pelo teste SNK na linha horizontal.

Legenda: $\mathrm{ES}$ = estacionamento, $\mathrm{BD}=$ borda do fragmento, $\mathrm{CFF}=$ centro do fragmento de floresta.

As maiores médias foram encontradas no estacionamento, provavelmente por existir poucas barreiras, aumentando assim a velocidade do vento (TONG et al., 2017), apesar do local ter prédios ao redor, no meio do estacionamento a densidade de possíveis barreiras para a quebra da corrente de ar é menor que no centro do fragmento. Para Oke et al. (2017) a explicação está na rugosidade encontrada na vegetação, pois a natureza porosa das árvores causa mudanças suaves na velocidade do vento, enquanto os edifícios criam alta diferença de pressão entre as direções de entrada da corrente de ar (barlavento) e saída (sotavento).

Em outros trabalhos feitos em fragmentos florestais situados em Curitiba, como Viezzer et al. (2015) e Martini e Biondi (2015) é possível constatar que a velocidade do vento é uma variável difícil de avaliar a influência microclimática, devido aos ventos próximos do solo e as características da estrutura urbana. Entretanto, no presente estudo é possível afirmar que, o fragmento de floresta reduz significativamente a velocidade do vento, aproximadamente $1 \mathrm{~m} / \mathrm{s}$, em todas as estações do ano. 


\section{CONCLUSÃO}

O fragmento de floresta urbana (Capão do Tigre) influencia o microclima imediato em todas as estações do ano, sendo que as maiores diferenças significativamente são verificadas entre o centro do fragmento e o estacionamento. A temperatura do ar foi a variável que melhor refletiu as diferentes características dos locais de coleta nas quatro estações do ano, demonstrando a influência da vegetação.

A conservação das florestas urbanas contribui para o bem estar social, biológico, ecológico.

\section{AGRADECIMENTOS}

Ao CNPq - Conselho Nacional de Desenvolvimento Científico e Tecnológico pelo financiamento desta pesquisa por meio da concessão da bolsa de Iniciação Científica.

\section{REFERÊNCIAS}

ALVES, R. S.; FIALHO, E. S. Clima urbano. Revista geografares, Espírito Santo, v.1, n.10, p.0816, 2012.

BARBIRATO, G. M.; SOUZA, L. C. L.; TORRES, S. C. Clima e cidade: a abordagem climática como subsídio para estudos urbanos. 1를ição. Maceió/AL: Edufal, 2007.

BARGOS, D. C.; MATIAS, L. F. Áreas verdes urbanas: um estudo de revisão e proposta conceitual. Revista da Sociedade Brasileira de Arborização Urbana, Piracicaba, v.6, n.3, p.172-188, 2011.

BIONDI, D.; BATISTA, A. C.; MARTINI, A.; GRISE, M. M. O efeito microclimático do bosque Capão da Imbuia na cidade de Curitiba-PR, Brasil. In: CONGRESSO FLORESTAL LATINOAMERICANO, 5., 2011, Lima. Anais... Lima: [s.n.], 2011. Não paginado.

CLIMATE-DATA. Clima Curitiba. Disponível em: <https://pt.climate-data.org/america-dosul/brasil/parana/curitiba-2010/>. Acesso em: 07 ago. 2019.

FEITOSA, S. M. R.; GOMES, J. M. A.; NETO, J. M. M.; ANDRADE, C. S. P. Consequências da urbanização na vegetação e na temperatura da superfície de Teresina - Piaui. Revista da Sociedade Brasileira de Arborização Urbana, Piracicaba, v.6, n.2, p.58-75, 2011.

GUIRAO, A. C.; FILHO, J. T. Preservação de um fragmento florestal urbano- estudo de caso: a ARIE mata de santa genebra, Campinas - SP. GEOUSP - Espaço e Tempo, São Paulo, v.15, n.1, p.147-158, 2011.

KUDO, S. A.; PEREIRA, H. S.; SILVA, S. C. P. A proteção jurídica dos fragmentos florestais urbanos: um estudo da paisagem e da legislação ambiental e urbanística da cidade de Manaus.

DEMA Desenvolvimento e Meio Ambiente, Manaus, v.38, p.521-540, 2016. 
LEAL, L.; MARTINI, A.; BIONDI, D.; BATISTA, A. C. Levantamento meteorológico expedito para análise da influência microclimática do Bosque Estadual João Paulo II, Curitiba - PR. In: ENCONTRO SUL-BRASILEIRO DE METEOROLOGIA, 4., 2011, Pelotas/RS. Anais... Pelotas: SBMET, 2011. p. 1-9.

MARTELLI, A.; JR, A. R. S. Arborização Urbana do município de Itapira - SP: perspectivas para educação ambiental e sua influência no conforto térmico. REGET/UFSM, Itapira, v.19, n.2, p.1018-1031, 2015.

MARTINI, A.; BIONDI, D. Microclima e conforto térmico de um fragmento de floresta urbana em Curitiba, PR. Floram Floresta e Ambiente, Curitiba, v.22, n.2, p.182-193, 2015.

MIGUEZ, L. A. L. Mapeamento e Monitoramento dos Maciços Vegetais do Município de Curitiba - PR. IPPUC - Banco de dados, Curitiba, 2001.

OKE, T. R.; MILLS, G.; CHRISTEN, A.; VOOGT, J. A. Urban Climates. 1ª Edição. Reino Unido - Cambridge: Cambridge University Press, 2017.

RONDON NETO, R. M.; KOZERA, C.; ANDRADE, R. R.; CECY, A. T.; HUMMES, A. P.; FRITZSONS, E.; CALDEIRA, M. V. W.; MACIEL, M. N. M.; SOUZA, M. K. F. Caracterização florística e estrutural de um fragmento de floresta ombrófila mista, em Curitiba, PR - Brasil. FLORESTA, Curitiba, v. 32, n. 1, p. 3-16, 2002.

SAMPAIO, A.; FARIA, M. C. Atualidades: 200 questões comentadas. $1^{a}$ edição. Rio de Janeiro: Clube de autores, 2014.

SANTOS, M. Urbanização concentrada e metropolização. In: SANTOS, M. (Ed). A urbanização brasileira. São Paulo: Editora da Universidade de São Paulo, p.77-91. 2008.

SILVA, D. A.; BIONDI, D.; MARTINI, A.; VIEZZER, J. Influência microclimática do bosque gutierrez na cidade de Curitiba - PR, Brasil. Enciclopédia Biosfera, Goiânia, v.10, n.19, p.2327-2338, 2014.

SOARES, R. V.; BATISTA, A. C.; TETTO, A.F. Meteorologia e Climatologia florestal. Curitiba: Os autores, 2015.

SOLDERA, C.; BATISTA, D. B.; MARTINI, A. Microclima e conforto térmico na trilha do Capão do Tigre - espaço com atividades de educação ambiental. Enciclopédia Biosfera, Goiânia, v.10, n.19, p.2348-2359, 2014.

TONG, S.; WONG, N. H.; TAN, C. L.; JUSUF, S. K.; IGNATIUS, M.; TAN, E. Impact of urban morphology on microclimate and thermal comfort in northern China. Solar Energy, Singapura, v.155, p.212-223, 2017.

VIEZZER, J.; BIONDI, D.; MARTINI, A.; SILVA, D. A. O benefício microclimático proporcionado pela Praça Alfredo Andersen na cidade de Curitiba-PR. Ciência e Natura, Santa Maria, v.37, Ed. Especial, p.138-143, 2015.

WANG, Y.; BAKKER, F.; DE GROOT, R.; WORTCHE, H.; LEEMANS, R. Effects of urban trees on local outdoor microclimate: synthesizing field measurements by numerical modelling. Urban Ecosystems, Olanda, v.18, n.4, p.1305-1331, 2015.

ZHANG, Z.; LV, Y.; PAN, H. Cooling and humidifying effect of plant communities in subtropical urban parks. Urban Forestry and Urban Greening, Amsterdam, v.12, n.3, p.323-329, 2013. 